Benedicto-López, Patricia \& Rodríguez-Cuadrado, Sara (2019). Discalculia: manifestaciones clínicas, evaluación y diagnóstico. Perspectivas actuales de intervención educativa. RELIEVE, 25(1), art. $7 . \quad$ doi: http://doi.org/10.7203/relieve.25.1.10125

Revista ELectrónica de Investigación y EValuación Educativa
RELIEVE

ISSN: 1134-4032
e-Journal of Educational Research,

Assessment and Evaluation

\title{
Discalculia: manifestaciones clínicas, evaluación y diagnóstico. Perspectivas actuales de intervención educativa
}

\author{
Dyscalculia: Clinical manifestations, evaluation and diagnosis. Current Perspectives of \\ educational intervention
}

\section{Benedicto-López, Patricia ${ }^{(1)}$ \& Rodríguez-Cuadrado, Sara ${ }^{(2)}$}

(1) Universitat Oberta de Catalunya $\quad$ (2) Universidad Autónoma de Madrid

\begin{abstract}
Learning difficulties and learning disorders are very frequent in schools nowadays, and they have been increasing in the field of mathematics. Such difficulties tend to be associated with other disorders such as dyslexia or Attention Deficit Hyperactivity Disorder (ADHD). The present article aims at deepening our understanding, definition and detection of dyscalculia, along with reviewing current educational treatments. Since 1990, concerns about learning difficulties in the classroom have been made explicit in policies such as the Spanish Law on the General Ordering of the Education System (LOGSE). Likewise, the indicators, symptoms, and prevalence of dyscalculia have been gathered since the publication of the DSM-IV and the ICD-10. Nonetheless, its detection, identification and intervention are below expectation. Features such as the knowledge of its underlying neurobiological basis are fundamental for a posterior psychopedagogical approach. We conclude, first, that we can only rely on a few specific tools for its detection. Second, we highlight the relevance of the detection of its risk factors. Finally, regarding current perspectives of treatment, early detection and the interdisciplinary nature of the interventions are supported
\end{abstract}

Keywords: Learning difficulties, dyscalculia, Early years education, Primary education, Secondary education, detection, educational intervention.

\begin{abstract}
Resumen
Actualmente, son numerosas las dificultades y trastornos del aprendizaje en el ámbito escolar, con un incremento en el área de las matemáticas. Tales dificultades suelen aparecer asociadas a otros trastornos como la dislexia o el Trastorno por Déficit de Atención e Hiperactividad (TDAH). Este artículo tiene como objetivo profundizar en la definición y detección de la discalculia, así como revisar las perspectivas actuales para su tratamiento desde la educación. Desde 1990 se explicita la problemática de las Dificultades de Aprendizaje en el aula con la publicación de la Ley de Ordenación General del Sistema Educativo español (LOGSE). Así mismo, desde la publicación del DSM-IV y el CIE-10, se han recogido los signos, síntomas y prevalencia de la discalculia. Sin embargo, su detección, identificación e intervención están por debajo de lo deseado. Aspectos como el conocimiento de las bases neurobiológicas subyacentes son fundamentales para el posterior abordaje psicopedagógico. Concluimos, en primer lugar, que existen muy pocos instrumentos específicos para la detección. En segundo lugar, destacamos la importancia de la detección de sus factores de riesgo. Finalmente, respecto a las perspectivas actuales de tratamiento se aboga por la detección temprana y el carácter interdisciplinar de las intervenciones.
\end{abstract}

Reception Date 2017 April 26

Approval Date 2019 April 04

Publication Date: 2019 April 15

Fecha de recepción 2017 April 26

Fecha de aprobación 2019 Abril 04

Fecha de publicación 2019 Abril 15

Palabras clave: Dificultades de aprendizaje, discalculia, Educación Infantil, Educación Primaria, Educación Secundaria, detección, intervención educativa.

Muchos/as niños/as son incapaces de clasificar objetos por su tamaño, emparejarlos, comprender el lenguaje aritmético o asimilar el concepto de cálculo racional (Gracia-Bafalluy \& Escolano-Pérez, 2014). Distintos autores señalan que es en la materia de matemáticas donde se encuentran uno de los índices de suspensos más altos 
(Fernández \& Llinares, 2014; Sans, LópezSala, Colomé, Boix \& Sanguinetti, 2013). La presente revisión tiene como objetivo clarificar y delimitar qué se entiende por discalculia, así como presentar sus tipos, manifestaciones clínicas, bases neurológicas y evaluación, incluyendo también las perspectivas actuales en la intervención educativa.

Los trastornos del aprendizaje fueron descritos por primera vez a finales del siglo XIX centrándose en la lectura y reconociendo su independencia del nivel de inteligencia, la instrucción, motivación y déficit sensoriales (Carboni-Román, Del Río Grande, Capilla \& Maestú, 2006). En 1962, Kirk y Bateman acuñan el término "Dificultades de Aprendizaje" (DA):

una dificultad de aprendizaje se refiere al retraso, trastorno o desarrollo retrasado en uno o más de los procesos del habla, lenguaje, lectura, escritura, aritmética u otros dominios escolares resultado de un hándicap psicológico causado por una posible disfunción cerebral y/o trastornos emocionales y conductuales. No es el resultado de retraso mental, deprivación sensorial 0 factores estructurales 0 instruccionales (p.14)

Desde esta perspectiva, habilidades tales como las lingüísticas, visoespaciales, de razonamiento y matemáticas forman parte de las DA. En 1978 la Secretaría de Educación del Reino Unido publica el Informe Warnock, que introduce el término "Necesidades Educativas Especiales" (NEE) sustituyendo al término "Educación Especial" (EE) (SEN, 1978). Este informe propone cambiar a un modelo de integración donde los niños deberán ser educados, preferentemente, en escuelas ordinarias, suponiendo un cambio radical en la consideración de la EE (Coronado Hijón, 2008).

Desde este momento, y con la publicación del DMS-IV (APA, 1993), se obtiene relativo consenso sobre los rasgos definitorios del alumno con DA. En primer lugar, su dominio estará por debajo de lo esperado para edad, nivel intelectual, capacidad y experiencias de aprendizaje significativas. En segundo lugar, las dificultades se observarían en una o más de estas siete áreas: expresión oral, comprensión auditiva, expresión escrita, lectura mecánica, comprensión lectora, cálculos matemáticos y razonamiento matemático. Además, la DA se relacionaría con otras dificultades en procesos psicológicos básicos como atención, memoria, percepción auditiva, percepción visual, coordinación visomotora y lenguaje oral. Finalmente, tales dificultades no se explican por déficits visuales o auditivos, déficit motóricos, retraso mental, trastorno emocional, deprivación económica, ambiental o cultural (APA, 1995, 2003, 2013). En la última versión del DSM (DSM-5, 2013), se incluye el trastorno específico del aprendizaje dentro de la categoría diagnóstica de trastornos del desarrollo neurológico. Dada la variedad de procesos que se pueden ver afectados, y la alta comorbilidad de los trastornos, es por ello fundamental establecer un diagnóstico diferencial (Millá, 2006; Serra-Grabulosa, 2014). Su carácter es crónico, y las personas que lo padecen suelen presentar otras problemáticas no sólo a nivel cognitivo (Gross-Tsur, Manor, \& Shalev, 1996), sino emocional (Artigas-Pallarés, 2002; Serra-Grabulosa, 2014).

En la literatura científica existen numerosos estudios en torno a las DA, especialmente en el caso de la dislexia y en el TDAH (ArtigasPallarés, 2002; Balaguer \& Tárraga, 2014; González-Castro et al., 2014; Martínez Zamora, Henao López \& Gómez, 2009). Sin embargo, otros como el "Trastorno del Aprendizaje No Verbal” (TANV) y la discalculia han sido menos estudiados (González-Castro et al, 2014). En España, la publicación del Informe Warnock (SEN, 1978) impulsa un modelo de EE articulado en la Ley 13/1982 de Integración Social del Minusválido que concreta el derecho constitucional de la educación e integración de los individuos con discapacidades o minusvalías. Este modelo establece los principios de normalización, sectorización, integración e individualización, tanto desde un punto de vista social como educativo, al 
tiempo que marca directrices acordes con los derechos establecidos a nivel constitucional y el Real Decreto 334/85 sobre ordenación de la EE. Ambas leyes contemplan las DA como una subcategoría de otra más amplia, las NEE (Coronado Hijón, 2008).

En 1990, con la publicación de la Ley de Ordenación General del Sistema Educativo (LOGSE) se establecen unos fines educativos generales para todo el alumnado $y$ se prescribe la adecuación de las enseñanzas a las características del alumnado con o sin NEE (LOGSE, art. 2.5). Posteriormente, la Ley Orgánica de Educación (LOE, 2006) y actualmente la Ley Orgánica de Mejora de la Calidad Educativa, (LOMCE, 2013) engloba al alumnado con DA dentro del término "Dificultades Específicas de Aprendizaje" (DEA). Este hecho supone entender las DEA como aquellas que surgen de la interacción entre alumno y contexto de enseñanza/aprendizaje, donde la escuela ha de dar una respuesta educativa eficaz desde el currículo teniendo en cuenta la diversidad del alumnado (Mora \& Aguilera, 2000).

\section{Hacia una definición de la discalculia}

La pérdida de la capacidad de realizar tareas de cálculo resultantes de una patología cerebral se conoce como acalculia (Ardilla \& Rosselli, 2002). La discalculia es una dificultad específica para el aprendizaje de la aritmética independiente de la inteligencia y la instrucción, con base neurobiológica y tentativamente genética (Sans et al., 2013). Lewandowsky y Stadelmann publicaron en 1908 por primera vez informes de trastornos del cálculo diferenciándolos del lenguaje. En 1925, Henschen acuña el término acalculia para explicar la pérdida de capacidad para el cálculo consecuencia del daño cerebral. Esto le llevó a proponer un sustrato anatómico para las operaciones aritméticas diferenciado al del lenguaje y la habilidad musical (Ardila \& Rosselli, 2002). En 1926, Berger introdujo la distinción entre acalculia primaria y secundaria, lo cual reconoce que los trastornos del cálculo pueden llevar asociadas a otras alteraciones verbales, espaciotemporales o de razonamiento. En 1940, Gerstmann propuso que la acalculia primaria estaba asociada con agrafia, desorientación derecha-izquierda, y agnosia digital conformando un síndrome neurológico conocido como "síndrome de Gerstmann."

En 1974, Kosc propuso el término discalculia por primera vez. Definió la discalculia como un trastorno diferenciado de otras alteraciones matemáticas, destacando su heradabilidad y/o afección congénita del sustrato cerebral responsable de las funciones matemáticas. Así mismo, establece distintas tipologías (ver Tabla 1).

Tabla 1. Tipologías de la discalculia (adaptado de Kosc, 1974)

\begin{tabular}{ll}
\hline \multicolumn{1}{c}{ TIPOLOGÍA } & \multicolumn{1}{c}{ DIFICULTAD } \\
\hline Verbal & Nombrar cantidades, números, términos, símbolos y relaciones. \\
Practognóstica & Enumerar, comparar y manipular objetos matemáticamente. \\
Léxica & Lectura de símbolos matemáticos. \\
Gráfica & Escritura de símbolos matemáticos. \\
Ideognóstica & Operaciones mentales y en la comprensión de conceptos matemáticos. \\
Operacional & Ejecución de operaciones y cálculos numéricos. \\
\hline
\end{tabular}

Dentro de las aportaciones iniciales, Badian (1983) encontró que los niños con discalculia muestran frecuentes dificultades numéricas de tipo espacial, en el cálculo y atencionalessecuenciales con escasa incidencia de alexia y agrafía para los números (Ardila, Rosselli \& Matute, 2005). Posteriormente, Geary
(1993) estableció una clasificación de la discalculia basada en tres tipos de errores: 1 ) visoespaciales, 2) de memoria semántica, y 3) de procedimiento. Rourke (1993) distinguió entre discalculia asociada con problemas de lenguaje y asociada a dificultades espacio-temporales, con 
problemas secuenciales e inversión de números (Spreen \& Strauss, 1995). En la actualidad, el término discalculia se usa de diferentes formas, desde como una dificultad del aprendizaje de las matemáticas o la aritmética, a una dificultad en el cálculo (Rebollo \& Rodríguez, 2006). Por tanto, pese a existir cierto consenso en cuanto a criterios diagnósticos, todavía se encuentran discrepancias en su definición, lo cual a su vez dificulta su evaluación e intervención.

Siguiendo el DSM-IV-TR (APA, 2000), la discalculia se define como un trastorno del aprendizaje en el que se observa una capacidad aritmética sustancialmente por debajo de lo esperado según la edad cronológica, el cociente intelectual y el nivel de escolaridad, donde tales dificultades interfieren significativamente en el rendimiento académico. En su última versión, el DSM-5 (APA, 2013), los trastornos del aprendizaje pasan a ser una subcategoría más general, los trastornos específicos del aprendizaje. Dentro del CIE10 (OMS, 1992), la discalculia se encuentra dentro de los trastornos específicos del desarrollo del aprendizaje escolar. Se refiere a un trastorno específico del cálculo, donde existe una alteración específica de la capacidad de aprendizaje de la aritmética no explicable por desórdenes del desarrollo neurológico o por una escolaridad inadecuada. Dentro del más reciente CIE-11 (OMS, 2018), la cual entrará en vigor en 2022, la discalculia deja de estar clasificada como un desorden de aprendizaje escolar (esto ocurre también para casos como el de la lectura) para recogerse como un trastorno evolutivo del aprendizaje con dificultades en matemáticas, dentro de la clasificación general de trastornos evolutivos del aprendizaje. Pese a compartir rasgos de exclusión con el CIE-10 (OMS, 1992), añade que tampoco puede ser explicado por falta de competencia en el idioma de la instrucción o por adversidades psicosociales. Sea como fuere, tanto el DMS como el CIE, ponen de manifiesto la necesidad de utilizar como instrumentos de evaluación pruebas estandarizadas de cálculo o de razonamiento.
El origen de la discalculia tampoco se ha establecido de forma clara. Rourke (1989) considera que es un trastorno cerebral determinado genéticamente mientras que autores como Fergusson, Horwood, y Lawton (1990) proponen que las causas están en el entorno del niño y el contexto social. En la acalculia, en contraposición a la discalculia, el trastorno matemático es consecuencia de una lesión cerebral. Sin embargo, la disfunción cognitiva de los procesos que subyacen a ambas podrían ser las mismas ya que los errores que presentan las personas que las padecen son similares.

Desarrollo normal de la habilidad para el cálculo.

La manipulación de cantidades es una capacidad innata que nos permite cuantificar todo aquello que tenemos a nuestro alrededor (Ardila \& Rosselli, 2002; Barrachina, SerraGrabulosa, Soler Vilageliiu \& Tolchinsky, 2014; Sans et al., 2013). A lo largo de la historia reciente, diferentes informes (Calpadi \& Miller, 1988; Koehler, 1951; Mechner, 1958) han argumentado que ciertos animales pueden utilizar conceptos numéricos y realizar operaciones aritméticas al menos hasta a una cierta cantidad. Sin embargo, las respuestas dadas son aproximadas (Dehaene, 1997). Este reconocimiento de cantidades se encuentra también en niños pequeños (Fuson, 1988; Wynn, 1990, 1992). Según Geary (2004), las competencias básicas se clasifican en habilidades cuantitativas innatas como la numerosidad, el pre-conteo y la ordinalidad, y competencias aritméticas adquiridas, desarrollas con posterioridad sobre las innatas, como son el número y el conteo. Hablamos, por tanto, de una capacidad básica que aparece en las primeras etapas del desarrollo tal y como mantiene Piaget, (1959) donde el desarrollo del pensamiento matemático tiene lugar durante la etapa de Educación Primaria (ver Lievegoed, 1999). Los bebés humanos son capaces de reconocer entre 3 y 6 artículos (Antell \& Keating, 1983), pero la capacidad de construir correspondencias entre número y 
cantidad emerge durante el segundo año de vida (Langer, 1986). El niño comienza a utilizar algunos nombres para los números, $\mathrm{y}$ se desarrolla la capacidad para contar hasta 3. De este modo se adquieren: el principio de correspondencia (cada objeto en una colección es emparejado con sólo un nombre de número) y el principio de orden estable (cada objeto tiene un nombre asignado una posición ordinal permanente en la lista). El principio de cardinalidad (el nombre del número final utilizado en una secuencia de conteo representa al conjunto) se observa en los niños de 3 años de edad (Gelman \& Meck, 1983), donde pueden contar por lo general hasta 10. Durante este período, el niño también está aprendiendo cómo funciona el sistema numérico y la memorización de las palabras de los números (Ardila \& Rosselli, 2002).

El primer y el segundo curso de Primaria se caracterizan por el manejo de la suma y la resta, utilizando los procedimientos de conteo con los dedos, en voz alta, y de memorización de sumas y restas para pequeñas cantidades (Ardila \& Rosselli, 2002). De tercero a quinto de Primaria manipulan los principios de la multiplicación y la división. De los 10 años a los 13 años, el uso del conteo con los dedos va desapareciendo, manteniéndose el conteo en voz alta (Grafman,1988; Siegler, 1987).

\section{Método}

\section{Objetivos de revisión}

Se pretende revisar la literatura científica relevante sobre aspectos tales como definición y tipos de discalculia, prevalencia y bases neurobiológicas, herramientas de evaluación y detección, y perspectivas de tratamiento actuales.

\section{Términos de búsqueda}

Discalculia (dyscalculia), aprendizaje matemático, trastornos del aprendizaje, dificultades en el aprendizaje de las matemáticas, intervención e identificación, trastornos de las matemáticas, trastornos del cálculo y la aritmética, dificultades tempranas en matemáticas, bases neurológicas de la discalculia, acalculia, procesamiento numérico, intervención didáctica, neuropsicología del desarrollo, detección precoz, atención temprana en las dificultades matemáticas y competencia matemática.

3. Criterios de inclusión/exclusión.

a. Fecha de publicación entre 2000 y 2018, incluyendo manuales y artículos anteriores que consideramos imprescindibles.

b. Se han incluido artículos en habla inglesa y castellana.

c. Se ha adoptado una perspectiva escolar (Educación Infantil, Primaria y Secundaria).

d. No se han incluido estudios relacionados con adultos.

e. Únicamente se han incluido artículos de índole científica y no de carácter divulgativo.

f. Se ha hecho uso de fuentes primarias como artículos originales y tesis, y secundarias como bases de datos.

\section{Estrategias de búsqueda}

Se han consultado las siguientes bases de datos y fuentes documentales: PubMed, Dialnet, ERIC, Google Académico, Revista de Neurología y PsycINFO.

\section{Estrategia para seleccionar estudios.}

Una vez elegidas las palabras clave, se seleccionaron los artículos en base a los criterios especificados anteriormente. Los contenidos de esta revisión se relacionan directamente con el objetivo general definido al inicio del método. Como se puede ver en la Tabla 2 (y en la Tabla 3 más abajo), la mayoría de los artículos consultados se centran en investigación básica, seguido por evaluación y diagnóstico, y tratamiento. El tema específico- 1 se refiere a artículos en los que se tratan de forma más acotada los trastornos del cálculo. Finalmente, el tema específico-2 menciona la discalculia de forma secundaria. 
Tabla 2. Referencias bibliográficas consultadas

\begin{tabular}{llcc}
\hline \multicolumn{1}{c}{ TEMA CENTRAL } & \multicolumn{1}{c}{ TEMA ESPECIFICO-1 } & TEMA ESPECIFICO-2 & $\mathrm{N}$ \\
\hline & Niños & 12 \\
\cline { 2 - 3 } & $\begin{array}{l}\text { Dificultades de aprendizaje y } \\
\text { Trastornos del aprendizaje }\end{array}$ & 7 \\
\cline { 2 - 3 } Investigación básica & Prevalencia y bases neurobiológicas & 9 \\
\cline { 2 - 3 } & $\begin{array}{l}\text { Modelos cognitivos y de } \\
\text { procesamiento de la información }\end{array}$ & 20 \\
\cline { 2 - 3 } & Comorbilidad & TDAH & 2 \\
\cline { 2 - 3 } & Psicología educativa & Dislexia & 9 \\
\hline Evaluación y diagnóstico & & & 18 \\
\hline Tratamiento & & 94 \\
\hline TOTAL DE ARTÍCULOS REVISADOS & \\
\hline
\end{tabular}

A continuación, en la Tabla 3, se recogen las referencias bibliográficas explicitando aquellos trabajos de índole teórica o empírica y temática (investigación básica, evaluación y diagnóstico y tratamiento). Como puede observarse en la tabla la mayoría de los artículos consultados se centran en investigación básica, seguida por evaluación y diagnóstico, y tratamiento.

Tabla 3. Ficha bibliométrica de los artículos incluidos en la revisión

\begin{tabular}{|c|c|c|}
\hline Autores/año & Trabajo & Temática \\
\hline Alonso Cánovas (2009)* & Empírico & Investigación Básica \\
\hline Antell y Keating (1983) & Empírico & Investigación Básica \\
\hline Ardila y Rosselli (2002)* & Empírico & Investigación Básica \\
\hline Ardila, Rosselli y Matute (2005) & Empírico & Investigación Básica \\
\hline Arribas, Santamaría, Sánchez- Sánchez y Fernández- Pinto (2015)* & Empírico & Evaluación y Diagnóstico \\
\hline Artigas-Pallarés (2002)* & Empírico & Investigación Básica \\
\hline Ausubel (1968) & Teórico & Tratamiento \\
\hline Badian $(1983)^{*}$ & Empírico & Investigación Básica \\
\hline Balaguer Rodríguez y Tárraga Mínguez (2014) & Teórico & Investigación Básica \\
\hline Barrachina, Serra-Grabulosa, Soler Vilageliiu y Tolchinsky (2014)* & Teórico & Investigación Básica \\
\hline Berger (1926) & Empírico & Investigación Básica \\
\hline Boix et al. (2001)* & Teórico & Investigación Básica \\
\hline Bryant, Bryant, Gersten, Scammacca, y Chavez (2008)* & Empírico & Tratamiento \\
\hline $\begin{array}{l}\text { Bryant, Bryant, Porterfield, Dennis, Falcomata, Valentine, Brewer y } \\
\text { Bell (2014)* }\end{array}$ & Empírico & Tratamiento \\
\hline Butterworth, Reeve, Reynolds y Lloyd (2008)* & Empírico & Investigación Básica \\
\hline Butterworth y Yeo (2004)* & Empírico & Tratamiento \\
\hline Callaway (2013)* & Empírico & Tratamiento \\
\hline Calpadi y Miller (1988) & Empírico & Investigación Básica \\
\hline Carboni-Román, Del Río Grande, Capilla y Maestú (2006) & Empírico & Investigación Básica \\
\hline Cipolotti y Butterworth (1995)* & Empírico & Investigación Básica \\
\hline Cohen $(1971)^{*}$ & Empírico & Investigación Básica \\
\hline Coronado Hijón (2008) & Empírico & Investigación Básica \\
\hline Davis, Bryson y Hoy (1992) & Empírico & Investigación Básica \\
\hline Dehaene (1997) & Empírico & Tratamiento \\
\hline Dehaene y Cohen (1995)* & Empírico & Investigación Básica \\
\hline Dehaene, Molko, Cohen y Wilson (2004)* & Empírico & Investigación Básica \\
\hline Dowker (2005)* & Empírico & Tratamiento \\
\hline Elliot, Smith y McCulloch (1996)* & Empírico & Evaluación y diagnóstico \\
\hline Estévez Pérez, Castro Cañizares y Reigosa Crespo (2008)* & Empírico & Investigación Básica \\
\hline Faramarzi y Sadri (2014)* & Empírico & Tratamiento \\
\hline Fernández y Llinares (2014) & Teórico & Investigación Básica \\
\hline Fergusson, Horwood y Lawton (1990) & Empírico & Investigación Básica \\
\hline Fuson (1988) & Empírico & Investigación Básica \\
\hline Garrido (2014)* & Teórico & Tratamiento \\
\hline Geary (1993) & Empírico & Investigación Básica \\
\hline Geary (2004) & Empírico & Investigación Básica \\
\hline
\end{tabular}


Benedicto-López, Patricia \& Rodríguez-Cuadrado, Sara (2019). Discalculia: manifestaciones clínicas, evaluación y diagnóstico. Perspectivas actuales de intervención educativa. RELIEVE, 25(1), art. $7 . \quad$ doi: http://doi.org/10.7203/relieve.25.1.10125

Tabla 3. Ficha bibliométrica de los artículos incluidos en la revisión (Continuación)

\begin{tabular}{|c|c|c|}
\hline Autores/año & Trabajo & Temática \\
\hline Gelman y Meck (1983) & Empírico & Investigación Básica \\
\hline Gerstmann (1940) & Empírico & Investigación Básica \\
\hline Ginsburg y Baroody (1983)* & Empírico & Evaluación y diagnóstico \\
\hline González-Castro, Rodríguez, Cueli, Cabeza y Álvarez (2014) & Empírico & Investigación Básica \\
\hline Gracia-Bafalluy y Escolano-Pérez (2014) & Empírico & Investigación Básica \\
\hline Grafman (1988) & Empírico & Investigación Básica \\
\hline Grégoire, Nöel y Van Nieuwenhoven (2015)* & Empírico & Evaluación y diagnóstico \\
\hline Gross-Tsur, Manor y Shalev (1996) & Empírico & Investigación Básica \\
\hline Gillum (2014)* & Empírico & Investigación Básica \\
\hline Henschen (1925) & Empírico & Investigación Básica \\
\hline Jacubovich (2006)* & Empírico & Investigación Básica \\
\hline Käser et al. (2013)* & Empírico & Tratamiento \\
\hline Kirk y Bateman (1962)* & Empírico & Investigación Básica \\
\hline Koehler (1951) & Empírico & Investigación Básica \\
\hline Kosc (1974)* & Empírico & Investigación Básica \\
\hline Kucian et al. (2011)* & Empírico & Investigación Básica \\
\hline Langer (1986) & Empírico & Investigación Básica \\
\hline Lewandowsky y Stadelmann (1908) & Empírico & Investigación Básica \\
\hline Lewis, Hitch y Walker (1994) & Empírico & Investigación Básica \\
\hline Lievegoed (1999) & Teórico & Investigación Básica \\
\hline Loveland, Fletcher y Bailey (1990)* & Empírico & Investigación Básica \\
\hline Manga y Campos (1991)* & Empírico & Evaluación y diagnóstico \\
\hline Martínez Zamora, Henao López y Gómez (2009)* & Empírico & Investigación Básica \\
\hline McCloskey, Caramazza y Basili (1985)* & Empírico & Investigación Básica \\
\hline Mechner (1958) & Empírico & Investigación Básica \\
\hline Millá (2006)* & Empírico & Tratamiento \\
\hline Miranda Casas, Meliá de Alba, Marco, Roselló y Mulas (2006) & Empírico & Investigación Básica \\
\hline Mora y Aguilera (2000) & Teórico & Investigación Básica \\
\hline Nelwan, Vissers y Kroesbergen (2018)* & Empírico & Tratamiento \\
\hline Olea, Ahumada y Líbano (1986)* & Empírico & Evaluación y diagnóstico \\
\hline Palomino y Crespo (2005)* & Empírico & Evaluación y diagnóstico \\
\hline Pérez, Arias y Mateos (2000)* & Empírico & Evaluación y diagnóstico \\
\hline Piaget (1959) & Teórico & Investigación Básica \\
\hline Portellano, Mateos y Martínez-Arias (2012)* & Empírico & Evaluación y diagnóstico \\
\hline Räsänen, Salminen, Wilson, Aunio y Dehaene (2009)* & Empírico & Tratamiento \\
\hline Re, Pedron, Tressoldi y Lucangeli (2014)* & Empírico & Tratamiento \\
\hline Rebollo y Rodríguez (2006) & Empírico & Investigación Básica \\
\hline Rourke (1987) & Empírico & Evaluación y diagnóstico \\
\hline Rourke (1988) & Empírico & Evaluación y diagnóstico \\
\hline Rourke (1993) & Empírico & Investigación Básica \\
\hline Santamaría, Arribas, Pereña y Seisdedos (2005)* & Empírico & Evaluación y diagnóstico \\
\hline Sans, Boix, Colomé, López-Sala y Sanguinetti (2012)* & Teórico & Investigación Básica \\
\hline Sans, López-Sala, Colomé, Boix y Sanguinetti (2013)* & Teórico & Investigación Básica \\
\hline Serra-Grabulosa (2014)* & Teórico & Investigación Básica \\
\hline Siegler (1987) & Empírico & Investigación Básica \\
\hline Shalev, Auerbach y Gross-Tsur (1995) & Empírico & Investigación Básica \\
\hline Shalev, Weirtman y Amir (1988) & Empírico & Investigación Básica \\
\hline Spreen y Strauss (1991)* & Empírico & Evaluación y Diagnóstico \\
\hline Strang y Rourke (1985)* & Empírico & Evaluación y diagnostico \\
\hline Temple (1997)* & Empírico & Investigación Básica \\
\hline Thurstone $(1938)^{*}$ & Empírico & Evaluación y diagnostico \\
\hline Thurstone y Thurstone (2004)* & Empírico & Evaluación y diagnostico \\
\hline Vygotsky (1931) & Empírico & Tratamiento \\
\hline Weschler (2012)* & Empírico & Evaluación y diagnostico \\
\hline Wilson et al. (2006)* & Empírico & Tratamiento \\
\hline Wynn (1990) & Empírico & Investigación Básica \\
\hline Wynn (1992) & Empírico & Investigación Básica \\
\hline Yuste, Martínez y Galve (2005)* & Empírico & Evaluación y diagnostico \\
\hline
\end{tabular}

Nota: Los artículos marcados con un asterisco $(*)$ son resultado de la revisión bibliográfica con importancia central. Los artículos sin asterisco se han empleado como complemento informativo en la introducción/discusión. 


\section{Resultados}

Prevalencia y bases neurobiológicas de la discalculia

La prevalencia de las DA en la población escolar es de entre el 5\% y el 15\% (Sans et al., 2013), y de entre el 2,27\% y el 6,4\% para la discalculia (Estévez Pérez, Castro Cañizares \& Reigosa Crespo, 2008). Además, se encuentra aproximadamente un $25 \%$ de comorbilidad entre la discalculia y otras alteraciones del desarrollo (Gross-Tsur et al., 1996). La distribución por sexos ha sido objeto de controversia, aunque algunos autores consideran que la discalculia tiende a afectar a ambos sexos igualmente (Lewis, Hitch \& Walker, 1994; Gross-Tsur et al., 1996). Sin embargo, debemos ser cautos, ya que la discalculia ha sido mucho menos estudiada que otras DA y por lo tanto queda mucho por conocer (Ardila \& Rosselli, 2002).

Respecto a las bases neuroanatómicas, Cohen (1971) propuso que la problemática en aritmética se podría explicar por dificultades en memoria a corto plazo (Shalev, Weirtman \& Amir, 1988). La relación entre discalculia y dificultades en la atención también ha sido ampliamente documentada (Badian, 1983; Davis, Bryson \& Hoy, 1992, Shalev, Auerbach, \& Gross-Tsur, 1995; Strang \& Rourke, 1985). Diversos modelos han sido propuestos para explicar el procesamiento numérico y el cálculo y sus bases neurobiológicas. Entre ellos, destacamos el modelo cognitivo de McCloskey et al. (1985), el modelo multi-ruta de Cipolotti y Butterworth (1995), y el modelo del triple código de Dehaene y Cohen (1995).

McCloskey et al. (1985) proponen un modelo cognitivo del procesamiento numérico para explicar los errores que comenten los pacientes con acalculia. Temple (1997) utilizó este modelo para estudiar la discalculia. Es un modelo modular donde diferentes subcomponentes pueden verse alterados selectivamente como consecuencia de una lesión cerebral (ver Figura 1).

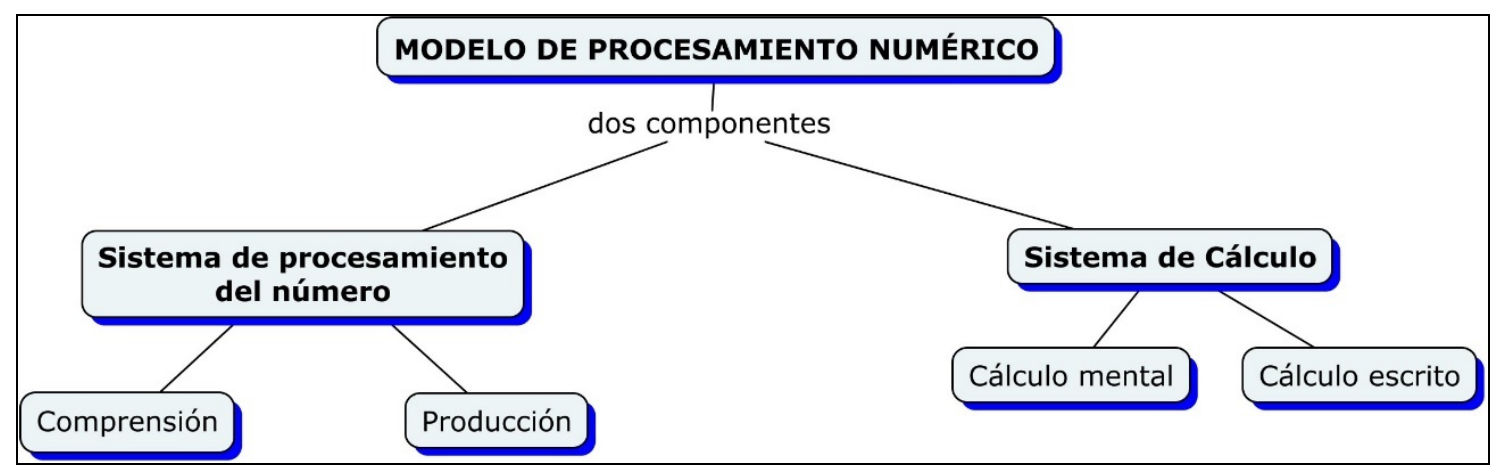

Figura 1. Modelo cognitivo de McCloskey et al. (adaptado de Jacubovich, 2006)

Consta de un sistema de procesamiento numérico con subsistema de entrada y subsistema de salida, los cuales proponen módulos separados para el procesamiento del código arábigo y del código verbal en sus modalidades fonológica (oral) y escrita (ortográfica). En segundo lugar, el modelo cuenta con un sistema de representaciones semánticas que ejerce de intermediario en la traducción de códigos de una entrada a una salida, mediante la codificación de magnitudes que actuaría de intermediario durante la resolución del cálculo aritmético (Serra-Grabulosa et al., 2010). Finalmente, encontramos un sistema de cálculo que se divide en dos subsistemas independientes, cálculo mental y cálculo escrito.

Cipolotti y Butterworth (1995), en su modelo multi-ruta extienden el modelo de McCloskey et al. (1985). Añaden rutas asemánticas que permiten la conversión entre unos códigos y otros sin acceder a la representación semántica del número. Se 
utilizaría una u otra ruta dependiendo de las demandas de la tarea, siendo inhibidas las rutas distintas a la activada. Por su parte, Dehaene y Cohen (1995) desarrollaron el modelo del triple código, constituido por tres módulos de información numérica mentalmente manipulables. Proponen tres hipótesis funcionales. Uno, que la información numérica se puede manipular en tres códigos: analógico (región parietal inferior a nivel bilateral), verbal-auditivo (áreas perisilvianas del hemisferio izquierdo) y arábigo (circunvolución fusiforme de ambos hemisferios). Dos, que la información se puede traducir de un código otro mediante rutas asemánticas. Tres, que la elección de un código u otro depende del tipo de operación mental que se realice. Con la ayuda de técnicas de neuroimagen en participantes sanos, Dehaene y Cohen (1995) señalan que los circuitos neurales del procesamiento numérico se localizan principalmente en el lóbulo parietal, aunque haya otras regiones implicadas tales como la corteza prefrontal, la parte posterior del lóbulo temporal, la corteza cingulada y distintas regiones subcorticales (ver Serra-Grabulosa et al, 2010). Según este modelo, la destreza en aritmética es probablemente el resultado de un intercambio continuo de información entre las representaciones visual, verbal y cuantitativa de los números. Así, la discalculia podría originarse de varias maneras: mediante daño en alguno de los tres módulos, un desarrollo inadecuado, o que las conexiones entre módulos no se hayan desarrollado adecuadamente (Alonso Cánovas, 2009).

\section{Evaluación y diagnóstico}

Según Strang y Rourke (1985), los errores que se encuentran en los niños con discalculia pueden clasificarse en: 1) organización espacial de cantidades, 2) atención visual, 3) de procedimiento en aritmética, 4) motores en la grafía al escribir cantidades, 5) de juicio y razonamiento numérico, 6) de memoria de cantidades, y 7) perseverancia en la resolución de operaciones aritméticas y problemas numéricos. Las manifestaciones clínicas de la discalculia difieren en cuanto a edad y nivel de escolaridad (Sans, Boix, Colomé, Lopez \& Sanguinetti, 2012). En la etapa de Educación Infantil se observan dificultades a la hora de clasificar objetos por sus características, en la comprensión de los conceptos "más que” y “menos que”, en ordenar elementos por tamaño, en la correspondencia uno a uno, para contar hasta 10, o en copiar números arábigos (Sans et al., 2012). En Primaria, las dificultades se asocian a la numeración (Boix et al., 2001; Sans et al., 2012, Sans et al., 2013; Serra-Grabulosa, 2014). Estos alumnos, por tanto, fallan en aspectos como aprender la hora o a contar dinero, confunden signos matemáticos, realizan transposiciones en la escritura de cifras, tienen problemas para memorizar conceptos matemáticos y para entender la posición de los números, y apenas automatizan hechos aritméticos.

En Secundaria se encuentran dificultades para entender conceptos abstractos, poca habilidad para aplicar destrezas matemáticas, errores en los cálculos, escasas estrategias de resolución de problemas y falta de automatización de hechos aritméticos (Barrachina et al., 2014; Boix et al., 2001; Sans et al., 2012, Sans et al., 2013). También se encuentran dificultades neuropsicológicas asociadas, como en el análisis táctil de los objetos y deficiencias en la interpretación de expresiones faciales y emocionales (Rourke, 1987), prosodia inadecuada en el lenguaje verbal (Rourke, 1988) y dificultades en la interpretación de los acontecimientos no verbales (Loveland, Fletcher \& Bailey, 1990).

El diagnóstico de la discalculia (como el de otras DA) es clínico (APA, 2000, 2013). Esto requiere recoger datos de la historia clínica del alumno, así como la revisión de informes escolares y contacto con el servicio de orientación de la escuela. Paralelamente, se llevará a cabo una evaluación neuropsicológica a través de pruebas estandarizadas (ver Tabla 4), de cara a establecer cuáles son las dificultades, valorar las funciones cognitivas alteradas $\mathrm{y}$ preservadas, detectar la presencia de posibles déficits asociados y hacer un diagnóstico 
Benedicto-López, Patricia \& Rodríguez-Cuadrado, Sara (2019). Discalculia: manifestaciones clínicas, evaluación y diagnóstico. Perspectivas actuales de intervención educativa. RELIEVE, 25(1), art. $7 . \quad$ doi: http://doi.org/10.7203/relieve.25.1.10125

diferencial. Por tanto, esta evaluación incluirá pruebas que evalúen el cociente intelectual, los procesos lectoescritores y los procesos psicológicos básicos (Barrachina et al., 2014), dado que la capacidad de cálculo es multifactorial (Ardila et al., 2005). Todos estos datos se valorarán de cara al establecimiento de un tratamiento educativo lo más ajustado posible. Actualmente, son pocos los instrumentos estandarizados para la detección y diagnóstico de los trastornos del cálculo en España, especialmente para Secundaria y Bachillerato. Algunos de los test para detectar y diagnosticar trastornos del cálculo en España se recogen en la siguiente tabla fruto de la revisión bibliográfica del presente trabajo (Tabla 4), donde es importante destacar que en ciertos casos (como en pruebas de aptitud académica y rendimiento intelectual) se suelen emplear subpruebas de escalas más generales (por ejemplo, en la WPPSI-IV).

Tabla 4. Pruebas estandarizadas para la evaluación de la discalculia

\begin{tabular}{|c|c|c|c|}
\hline PRUEBA & DENOMINACIÓN & BAREMO & AUTORES \\
\hline TEDI-MATH & $\begin{array}{l}\text { Test para el diagnóstico de las } \\
\text { Competencias Básicas en } \\
\text { Matemáticas }\end{array}$ & $\begin{array}{l}2^{\circ} \text { ciclo de } \\
\text { Infantil- } 3^{\circ} \text { de } \\
\text { Primaria }\end{array}$ & $\begin{array}{l}\text { Grégoire, Nöel, \& Nieuwenhoven, } \\
2015 \text { (adaptado por Sueiro \& Pereña, } \\
\text { 2015) }\end{array}$ \\
\hline TEMA-3 & $\begin{array}{l}\text { Test de Competencia } \\
\text { Matemática Básica }\end{array}$ & 3-8 años & $\begin{array}{l}\text { Ginsburg \& Baroody, } 1983 \text { (adaptado } \\
\text { por Núñez \& Lozano, 2007) }\end{array}$ \\
\hline Prueba de Cálc & o y Nivel Matemático & 7-12 años & Palomino \& Crespo, 2005 \\
\hline Prueba de Apti & d y Rendimiento Matemático & 7-12 años & Olea, Ahumada \& Líbano 1986 \\
\hline WPPSI-IV & $\begin{array}{l}\text { Escala de Inteligencia de } \\
\text { Weschler para Preescolar y } \\
\text { Primaria }\end{array}$ & 2-7 años & $\begin{array}{l}\text { Weschler, } 2012 \text { (adaptado por el Dpto } \\
\text { de I+D Pearson Clinical and Talent } \\
\text { Assessment, 2014) }\end{array}$ \\
\hline BADyG-R & $\begin{array}{l}\text { Batería de } \\
\begin{array}{l}\text { Diferenciales } \\
\text { Renovado. }\end{array}\end{array}$ & $\begin{array}{l}\text { De Infantil a } \\
\text { Bachillerato }\end{array}$ & Yuste, Martínez \& Galve, 2005 \\
\hline LURIA-DNI & $\begin{array}{l}\text { Batería de } \quad \text { Diagnóstico } \\
\text { Neuropsicológico Infantil } \\
\end{array}$ & 7-10 años & Manga \& Ramos, 1991 \\
\hline CUMANÍN & $\begin{array}{l}\text { Cuestionario de Madurez } \\
\text { Neuropsicológica Infantil }\end{array}$ & 3-6 años & Pérez, Arias \& Mateos, 2000 \\
\hline CUMANES & $\begin{array}{l}\text { Cuestionario de Madurez } \\
\text { Neuropsicológica Escolar }\end{array}$ & 7-12 años & $\begin{array}{l}\text { Portellano, Mateos \& Martínez- Arias, } \\
2012\end{array}$ \\
\hline BAS II & $\begin{array}{l}\text { Escalas de Aptitudes } \\
\text { Intelectuales }\end{array}$ & 2-17 años & $\begin{array}{l}\text { Elliot, Smith \& McCullouch, } 1996 \\
\text { (adaptada por Arribas y Corral, 2011) }\end{array}$ \\
\hline PMA & $\begin{array}{l}\text { Test de Aptitudes Mentales } \\
\text { Primarias }\end{array}$ & $\begin{array}{l}\text { A partir de } 10 \\
\text { años }\end{array}$ & Thurstone, 1938 \\
\hline TEA & Test de Aptitudes Escolares & 8-18 años & Thurstone \& Thurstone, 2004 \\
\hline BAT-7 & Batería de Aptitudes de TEA & $\begin{array}{l}\text { A partir de } 12 \\
\text { años }\end{array}$ & $\begin{array}{l}\text { Arribas, Santamaría, Sánchez-Sánchez } \\
\text { \& Fernández -Pinto, } 2013\end{array}$ \\
\hline EFAI & $\begin{array}{l}\text { Evaluación Factorial de las } \\
\text { Aptitudes Intelectuales }\end{array}$ & $\begin{array}{l}\text { A partir de } 7 \\
\text { años }\end{array}$ & $\begin{array}{l}\text { Santamaría, Arribas, } \\
\text { Seisdedos, } 2005\end{array}$ \\
\hline
\end{tabular}

Perspectivas actuales en la intervención educativa.

La atención a las DA debe ser objeto de detección e intervención tempranas para prevenir el posible fracaso escolar del alumnado (Millá, 2006). El niño con discalculia requiere una enseñanza más intensiva y explícita sobre el sentido numérico, más práctica en el uso del sistema numérico y experiencias concretas con números grandes y pequeños (SerraGrabulosa, 2014). El carácter marcadamente temprano en la detección de los problemas matemáticos sugiere la necesidad de instaurar programas de reeducacion individualizados durante la infancia, lo cual facilitará su eficacia (Serra-Grabulosa, 2014). Esta reeducación debe ser muy dirigida cumpliendo con los objetivos mínimos y estándares básicos del currículo escolar. Estos programas 
mejorarán el rendimiento académico del alumno en el área de matemáticas y en general, y lo harán sentir más competente (Boix, Colomé, Lopez \& Sanguinetti, 2013).

Para elaborar un programa de reeducación eficaz, los objetivos variarán en función de los conocimientos previos del alumno (Ausubel, 1968), situándoles en su Zona de Desarrollo Próximo (Vygotsky, 1931). Entre los objetivos de reeducación, destacamos: 1) comprender el valor de cada número y su relación con los otros, 2) entender la composición/descomposición de los números, 3) contar de manera precisa y flexible, 4) entender el uso de múltiplos de 10, 5) automatizar los aprendizajes, 6) aumentar la confianza con los números y las matemáticas y 7) aplicar lo aprendido a la vida real.

Barrachina et al. (2014) sugieren una serie de directrices de cara a una reeeducación efectiva. En primer lugar, potenciar el aspecto lúdico a fin de ofrecer las experiencias de éxito en niños que normalmente presentan fracaso escolar. Además, hay que reforzar positivamente al alumno de cara a aumentar la confianza. Es por ello que los objetivos han de plantearse a corto plazo, el trabajo con debe ser altamente estructurado y los materiales usados variados, potenciando la multisensorialidad (Serra-Grabulosa, 2014). Teniendo en cuenta la baja velocidad de procesamiento numérico del alumno, no debemos limitarnos por el tiempo y las sesiones tendrán que mantener una periodicidad mínima de dos sesiones semanales. El seguimiento del alumno se deberá realizar en todas las materias y las familias deberán implicarse en el tratamiento. Dar una respuesta educativa ajustada implica elaborar un Plan de Trabajo Individualizado y explicitar en él una Adaptación Curricular que se adapte las necesidades e intereses del alumnado, procurar aprendizajes significativos y funcionales, coordinar a todos los agentes implicados en el desarrollo del niño y adaptar el proceso de evaluación evitando perjudicar al alumno (Garrido, 2014).
Aunque limitadas, contamos con ciertas alternativas disponibles para la reeducación en discalculia y/o dificultades matemáticas. Por fortuna, tal y como señala Gillum (2014), recientemente podemos observar un interés creciente en el desarrollo de diferentes intervenciones para tratar la discalculia. El foco de tales intervenciones es variado. Puede tratar tanto el conocimiento de hechos matemáticos, la habilidad para llevar a cabo procedimientos matemáticos, o entender cómo usar principios matemáticos, entre otros (Dowker, 2005). Sin embargo, queremos enfatizar que lo primordial es la identificación de, y actuación sobre, los primeros signos de dificultades matemáticas (esto es, la intervención temprana). Las intervenciones pueden darse en forma de intervención en el aula (ya sea dentro del currículo educativo o de modo adicional) y pueden obedecer a un enfoque tradicional o mediante el uso de las Tecnologías de la Información y la Comunicación (TIC). En primer lugar, revisaremos aquellas propuestas de enfoque más "tradicional” (es decir, sin uso de TIC) para luego centrarnos en las TIC.

Butterworth y Yeo, pioneros en la investigación sobre discalculia, publicaron en 2004 Dyscalculia Guidance, un manual de enfoque lúdico para guiar la enseñanza de las matemáticas en Primaria tanto a nivel individual, como en grupo. El manual recoge propuestas para entender el sistema numérico y consolidar la línea numérica mental, trabajar con números grandes y mejorar estrategias de cálculo.

Re, Pedron, Tressoldi, y Lucangeli (2014) realizaron un exitoso entrenamiento individual a fin de mejorar el concepto de número, la automaticidad en recuperar y usar hechos aritméticos, cálculo mental y escrito. Otros enfoques proponen la intervención grupal durante el tiempo normal de clase, centrándose en mejorar el aprendizaje del concepto de número, operaciones y razonamiento cuantitativo (ver Bryant, Bryant, Gersten, Scammacca, \& Chavez, 2008; Bryant et al., 2014). Así mismo, podemos encontrar intervenciones que han adoptado un enfoque neuropsicológico en alumnos con discalculia. 
En el estudio de Faramarzi y Sadri (2014), la intervención se centraba en reforzar la memoria auditiva y visual, reforzar la atención, entrenar funciones ejecutivas, desarrollar la percepción visuoespacial y reforzar el lenguaje. Los estudiantes que completaron la intervención neuropsicológica mostraron mejoras significativas en su desempeño matemático en comparación con el grupo control. Otro estudio reciente (Nelwan, Vissers, \& Kroesbergen, 2018) implementó un entrenamiento en memoria de trabajo para niños con dificultades atencionales y/o matemáticas, donde un grupo recibió la intervención con un alto nivel de entrenamiento, otro grupo recibió la misma intervención con menos entrenamiento y el grupo control no recibió la intervención. Se tomaron medidas tanto de memoria de trabajo visual y verbal como de habilidades matemáticas. El grupo que recibió más entrenamiento mejoró más que el grupo con menos entrenamiento, en memoria de trabajo visual y habilidades matemáticas, y mantuvo la mejoría en matemáticas en el tiempo, mostrando la relevancia de la motivación y un buen entrenamiento en las intervenciones.

Centrándonos ahora en el uso de las TIC, estas potencian el aspecto lúdico del aprendizaje de las matemáticas. Algunos ejemplos son el software creado por Dehaene llamado The Number Race (2004), basado en su modelo cognitivo, y el Rescue Calcularis (2011) creado por Kucian et al. (2011). The Number Race es un programa diseñado principalmente para niños de entre 4 y 8 años dirigido a aprender conceptos matemáticos básicos y aritmética. El juego pretende fortalecer los circuitos cerebrales implicados en la manipulación y representación mental de los números (Dehaene, Molko, Cohen, \& Wilson, 2004; Wilson et al., 2006). En 2009, Räsänen, Salminen, Wilson, Aunio y Dehaene reportaron que Number Race mejoró la habilidad de 15 niños discalcúlicos en edad preescolar a la hora de realizar tareas de comparación de números, sin efecto en conteo o aritmética. El software Rescue Calcularis es un programa de entrenamiento basado en la computadora Calcularis (Kucian et al, 2011), diseñado sobre la base del desarrollo típico y atípico de las habilidades matemáticas. Las mejoras más destacadas se observaron en restas y representación numérica (Käser et al., 2013). Otra opción disponible es Number Sense, una página web desarrollada por el equipo de Butterworth y Laurillard en el Instituto de Educación de Londres (http://lownumeracy.ning.com), ver también Number Catcher

http://www.thenumbercatcher.com/nc/home.php

), la cual está siendo empleada por numerosas escuelas en Londres, extendiéndose a lo largo del mundo en países como Cuba, China y Singapur. Pese a que los juegos originales de Number Sense no están disponibles a fecha de 2019, la nueva web se actualiza con frecuencia, y en ella podemos encontrar enlaces a juegos nuevos (como "NumberBeads") y publicaciones de interés sobre la discalculia. La intención de los juegos incluidos en Number Sense es la de nutrir aquellas habilidades que están, según Butterworth, en las raíces de la cognición numérica y en el corazón de la discalculia: manipular cantidades precisas. Pese a los planes de evaluar el software mediante estudios controlados en el Centro de Neurociencias de Cuba, aún necesitamos artículos científicos que evalúen la efectividad de Number Sense (ver Callaway, 2013). Otros estudios, sin embargo, no han encontrado ventaja en la administración de intervenciones mediante TIC en comparación con la instrucción por maestros usando técnicas de modelado cognitivo con instrucciones explícitas y retroalimentación inmediata y correctiva (ver Leh \& Jitendra, 2013).

\section{Discusión}

A lo largo del presente artículo de revisión hemos recabado información relativa a la definición y tipos de discalculia, prevalencia y bases neurobiológicas subyacentes, herramientas e instrumentos para la detección de la discalculia y perspectivas actuales en la intervención educativa. Para ello, ha sido necesario profundizar en cuestiones tales como qué entendemos por DA y cuáles son sus características. Así, podemos decir que la discalculia es un trastorno crónico del área de 
las matemáticas, que afecta a todas las edades, siendo de vital importancia su detección y diagnóstico lo más tempranamente posible (Ardila \& Rosselli, 2002; Millá, 2006). En nuestro país, la Ley de Mejora de la Calidad Educativa, LOMCE (2013) y anteriormente la Ley Orgánica de Educación, LOE (2006), enmarcan la discalculia dentro de las DEA, considerando a los alumnos que padecen dicho trastorno como Alumnos con Necesidades Específicas de Apoyo Educativo (ACNEAE). Tales alumnos son susceptibles de beneficiarse de una serie de medidas educativas que no les aleje cada vez más del currículo ordinario y les proporcione una respuesta educativa eficaz y ajustada a sus necesidades.

Este trastorno del cálculo ha sido escasamente estudiado en comparación con otros trastornos del neurodesarrollo, como los clasifica actualmente, DSM-5 (2013). Sin duda, en la literatura científica existe un número mayor de artículos de investigación relativos a la dislexia o al TDAH. Pese a esto, encontramos algunos trabajos de dislexia y discalculia o discalculia y TDAH (ArtigasPallarés, 2002; Martínez Zamora et al., 2009; Miranda Casas, Meliá de Alba, Marco Taverner, Roselló \& Mulas, 2006), dada su comorbilidad. De ello, se deriva la necesidad de establecer unos criterios claros para la detección y diagnóstico diferencial de la discalculia. Hemos observado que el DSM, tanto en su cuarta como en su quinta edición (APA, 1994, 2000, 2013) ponen de manifiesto la necesidad y obligatoriedad de utilizar pruebas estandarizadas específicas. Pero de nuevo, nos encontramos con muy poca investigación al respecto lo que hace que no dispongamos de instrumentos específicos para el cálculo, por lo que nos vemos obligados a utilizar subpruebas de escalas más generales (Escalas Weschler (Weschler, 2012), Cuamanin (Pérez et al., 2010), PMA (Thurstone, 1938), EFAI (Santamaría et al. 2005), entre otras).

Un tema controvertido es el del Cociente Intelectual (CI) que como establecen los manuales diagnósticos DSM y la clasificación de la OMS, CIE-10 y CIE-11 (1992, 2018), ha de encontrarse dentro de la normalidad. Sin embargo, como ya sabemos, en la discalculia no solo está afectada el área de las matemáticas sino también la velocidad de procesamiento, las funciones ejecutivas e incluso las habilidades lingüísticas (Badian, 1983; Millá, 2006; Strang \& Rourke, 1985; Shalev et al., 1995) que en la mayoría de los test de inteligencia forman parte del CI total, como ocurre en las Escalas Weschler (Weschler et al., 2012). Este solapamiento de dificultades hace que el CI no sea, quizás, un adecuado criterio de exclusión. Paralelamente al uso de pruebas estandarizadas y al tratarse de un trastorno clínico, es necesaria una valoración clínica de tipo neuropsicológica. Es en este punto, además de la revisión del historial clínico será necesario profundizar en aspectos tales como la memoria de trabajo, la velocidad de procesamiento o las funciones ejecutivas que tan estrechamente relacionados están con el trastorno del cálculo que nos ocupa (Badian, 1983; Strang \& Rourke, 1985; Shalev et al., 1995).

Determinadas las causas de la dificultad y el perfil neuropsicológico, así como de los recursos (personales y materiales) con los que contamos, se procederá al establecimiento de un plan de intervención. La identificación temprana del trastorno del cálculo debe realizarse, a ser posible, en los primeros cursos de la Educación Primaria. Será en este momento cuando el equipo docente deberá desarrollar un programa de enseñanza de las matemáticas adaptado, basado en las dificultades encontradas con el fin de conseguir un nivel lo más próximo al resto de sus compañeros/as. La atención temprana produce importantes efectos a corto plazo en los niños de riesgo y en los que nacen con discapacidad al prevenir o minimizar los retrasos en el desarrollo (Millá, 2006). Este abordaje se realizará desde una perspectiva interdisciplinar tanto en el diagnóstico como en la intervención. De este modo, proponemos incidir en cuatro grandes ámbitos: a) sanitario: a nivel de neurólogo, neuropsicólogo, psicólogo y pediatra, fundamentalmente, b) social: muchos alumnos que sufren discalculia manifiestan niveles de autoestima bajos y a 
menudo, se encuentran muy frustrados, c) familiar: es necesaria una coordinación y compromiso de los padres en la reeducación de sus hijos y d) educativo: implementando recursos y programas individualizados (ver también Millá, 2006). Desde esta perspectiva, decimos que el alumno con discalculia presenta necesidades específicas de apoyo educativo asociadas a DEA. En el ámbito educativo destacamos la labor de los Equipos de atención temprana, los Equipos Orientación Educativa y Psicopedagógica y los Departamentos de Orientación. Pese a que actuar sobre estas dificultades representa una medida muy eficaz para reducir sus efectos sobre aprendizajes posteriores, no disponemos de protocolos al respecto.

Tal como recoge la actual Ley de Educación española (LOMCE, 2013), dar una respuesta educativa eficaz pasa por adaptar los elementos fundamentales que componen el currículo (art.6). Esto es, los objetivos de cada enseñanza y etapa educativa, competencias, o capacidades para aplicar de forma integrada los contenidos propios de cada enseñanza y etapa educativa, con el fin de lograr la realización adecuada de actividades y la resolución eficaz de problemas complejos, contenidos, o conjuntos de conocimientos, habilidades, destrezas y actitudes que contribuyen al logro de los objetivos de cada enseñanza y etapa educativa y a la adquisición de competencias, metodología didáctica, estándares y resultados de aprendizaje evaluables y criterios de evaluación del grado de adquisición de las competencias y del logro de los objetivos de cada enseñanza y etapa educativa.

En definitiva, podemos decir que, pese a contar con una larga historia en el tema de las DA, la terminología que las define y los criterios de diagnosis que actualmente manejamos todavía no están claros. Existen muy pocas herramientas e instrumentos específicos para la detección de la discalculia y los tratamientos educativos están todavía muy lejos de la realidad del aula, extremadamente compleja y diversa que requiere de una intervención multidisciplinar. El objetivo de nuestra revisión es ofrecer una visión general, aunque exhaustiva y científica, de un trastorno de aprendizaje tan desconocido como es la discalculia. Dada la escasez de estudios al respecto (sobre todo en comparación con otros trastornos del aprendizaje), aún quedan muchas incógnitas por resolver. Por ello, además de los déficits que hemos identificado en la actual revisión, consideramos que áreas relevantes para futuras investigaciones son la exploración de qué factores de riesgo están incidiendo en el desarrollo de este trastorno, por un lado, y por otro lado la elaboración de pruebas o test estandarizados que permitiesen detectar no sólo si un alumno presenta o no discalculia, sino la comorbilidad con otros trastornos poco conocidos como es el TANV.

\section{Referencias}

Alonso Cánovas, D. (2009). Cerebro, números y educación. Aula: Revista de Pedagogía de la Universidad de Salamanca, (15), 79-90.

American Psychiatric Association. (1995). DSM-IV. Manual diagnóstico y estadístico de los trastornos mentales. Barcelona: Masson.

American Psychiatric Association. (2003). DSM-IV-TR. Manual diagnóstico y estadístico de los trastornos mentales. Barcelona: Masson.

American Psychiatric Association. (2013). DSM-5. Manual diagnóstico y estadístico de los trastornos mentales. Editorial Médica Panamericana.

Antell, S. E. \& Keating, D. (1983). Perception of numerical invariance by neonates. Child Dev. 54, 695-701. doi: https://doi.org/10.2307/1130057

Ardila, A. \& Rosselli, M. (2002). Acalculia and dyscalculia. Neuropsychology review, 12(4), 179-231. doi:

https://doi.org/10.1023/A:1021343508573

Ardila, A., Rosselli, M. \& Matute, E. (2005). Neuropsicología clínica. México: Manual moderno.

Arribas, D., Santamaría, P., Sánchez- Sánchez, F. \& Fernández- Pinto, I. (2015). BAT7: Batería de aptitudes de TEA: manual. TEA.

Artigas-Pallarés, J. (2002). Problemas asociados a la dislexia. Revista de Neurología, 34(1), 7- 
Benedicto-López, Patricia \& Rodríguez-Cuadrado, Sara (2019). Discalculia: manifestaciones clínicas, evaluación y diagnóstico. Perspectivas actuales de intervención educativa. RELIEVE, 25(1), art. $7 . \quad$ doi: http://doi.org/10.7203/relieve.25.1.10125

\section{3.} https://doi.org/10.33588/rn.34S1.2002063

Ausubel, D. P. (1968). The Psychology of Meaningful Learning; an Introduction to School Learning. Grune and Stratton.

Balaguer Rodríguez, P. \& Tárraga Mínguez, R. (2014). Revisión de los síndromes que comportan dificultades de aprendizaje en las matemáticas. Orientaciones para la intervención educativa. Radiocrea 37 (3), 323-333.

Badian, N.A. (1983). Developmental dyscalculia. In: Mykelbost HR, Ed. Progress in learning disabilities. Grune and Stratton. New York.

Barrachina, L.A., Serra-Grabulosa, J.M., Soler Vilageliiu, O. \& Tolchinsky, L. (2014). Trastornos de aprendizaje de la escritura y las matemáticas. Barcelona: UOC.

Berger, H. (1926). Uber Rechenstorunger bei Herderkraunkunger des Grosshirns. Arch. Psychiatr. Nervenkr. 78, 236-263. doi: https://doi.org/10.1007/BF01996620

Boix, C., Colomé, R., López, A., Sanguinetti, A., Caro, M., y Sans, S. (2001). Los trastornos del aprendizaje. Unitat de Transtorns de l'Aprenentatge (UTAE). Servei de Neurologia de l'Hospital Sant Joan de Déu.

Bryant, D. P., Bryant, B. R., Gersten, R., Scammacca, N., \& Chavez, M. M. (2008). Mathematics Intervention for First- and Second-Grade Students With Mathematics Difficulties. Remedial and Special Education, 29(1), 20-32. doi: https://doi.org/10.1177/0741932507309712

Bryant, B. R., Bryant, D. P., Porterfield, J., Dennis, M. S., Falcomata, T., Valentine, C., Brewer, C., Bell , K. (2014). The Effects of a Tier 3 Intervention on the Mathematics Performance of Second Grade Students With Severe Mathematics Difficulties. Journal of Learning Disabilities, 49 (2), 1-13.

Butterworth, B., Reeve, R., Reynolds, F. \& Lloyd, D. (2008). Numerical thought with and without words: evidence from indigenous Australian children. Proc. Natl. Acad. Sci. USA, 105, 13179-84. doi: https://doi.org/10.1073/pnas.0806045105

Butterworth, B. \& Yeo, D. (2004). Dyscalculia guidance. London: NFER-Nelson.
Callaway, E. (2013). Dyscalculia: Number Games. Nature, 493(7431), 150-153. doi: https://doi.org/10.1038/493150a

Calpadi, E. J. \& Miller, D. J. (1988). Counting in rats: Its functional significance and the independent cognitive processes that constitute it. J. Exp. Psychol. Anim. Behav. Process, 14, 3-17. doi: https://doi.org/10.1037/00977403.14.1.3

Carboni-Román, A., Del Río Grande, D., Capilla, A. \& Maestú, T (2006). Bases neurobiológicas de las dificultades de aprendizaje. Revista de neurología, 42 (supl 2), 171-175. doi: https://doi.org/10.33588/rn.42S02.2005832

Cipolotti, L. \& Butterworth, B. (1995). Toward a multiroute model of number processing: Impaired transcoding with preserved calculation skills. Journal of Experimental Psychology: General, 124, 375-390. doi: https://doi.org/10.1037/0096-3445.124.4.375

Cohen, R. (1971). Dyscalculia. Arch. Neurol. 4, 301-307. doi: https://doi.org/10.1001/archneur.1961.004500 $\underline{90067010}$

Coronado Hijón, A. (2008). Dificultades de aprendizaje de las matemáticas: conceptos básicos y diagnóstico. Revista de Humanidades, 15, 237-252.

Davis, H., Bryson, S. \& Hoy, C. (1992). Case study of language and numerical disability: A sequential processing deficit? Annals of Dyslexia 42, 69-89. doi: https://doi.org/10.1007/BF02654939

Dehaene, S. (1997). The Number Sense. How the Mind Creates Mathematics. Oxford University Press, New York.

Dehaene, S. \& Cohen, L. (1995). Towards an anatomical and functional model of number processing. Mathematical cognition, 1(1), 83120.

Dehaene, S., Molko, N., Cohen, L., \& Wilson, A. J. (2004). Arithmetic and the brain. Current opinion in neurobiology, 14(2), 218-224. doi: https://doi.org/10.1016/j.conb.2004.03.008

Dowker, A. (2005). Early Identification and Intervention for Students With Mathematics Difficulties. Journal of Learning Disabilities, 38(4), 324-332. doi: https://doi.org/10.1177/002221940503800408 $\underline{01}$ 
Benedicto-López, Patricia \& Rodríguez-Cuadrado, Sara (2019). Discalculia: manifestaciones clínicas, evaluación y diagnóstico. Perspectivas actuales de intervención educativa. RELIEVE, 25(1), art. $7 . \quad$ doi: http://doi.org/10.7203/relieve.25.1.10125

Elliot, C. D., Smith, P. \& McCulloch, K. (1996). British ability scales II. Windsor. England: NFER-Nelson.

Estévez Pérez, N., Castro Cañizares, D. \& Reigosa Crespo (2008). Bases Biológicas de la Discalculia del desarrollo. Revista genética comunitaria, 2 (3), 14-19.

Faramarzi, S., \& Sadri, S. (2014). The effect of basic neuropsychological interventions on performance of students with dyscalculia. Neuropsychiatria i Neuropsychologia, 9(2), 48.

Fernández, C. \& Llinares, S. (2014). Dificultades en la enseñanza y el aprendizaje de las matemáticas. Dentro de Badia Garganté, A., et al. Dificultades de aprendizaje de los contenidos curriculares. (1 $1^{\text {a }}$ ed., p.25-101). Barcelona: UOC.

Fergusson, D. M., Horwood, L. \& J. Lawton, J. M. (1990). Vulnerability to childhood problems and family social background. $J$. Child Psychol. Psychiatry 31, 1145-1160. doi: $\quad$ https://doi.org/10.1111/j.14697610.1990.tb00852.x

Fuson, K. C. (1988). Children's Counting and the Concepts of Number. Springer-Verlag, New York. doi: https://doi.org/10.1007/9781-4612-3754-9

Garrido, J. (2014). Programación de actividades para la educación especial. Madrid: CEPE.

Geary, D. C. (1993). Mathematical disabilities: Cognitive, neuropsychological and genetic components. Psychol. Bull. 114, 345-362. doi: $\quad$ https://doi.org/10.1037/00332909.114.2.345

Geary, D. C. (2004). Mathematics and learning disabilities. Journal of learning disabilities, 37(1), 4-15. doi: https://doi.org/10.1177/002221940403700102 $\underline{01}$

Gelman, R. \& Meck, E. (1983). Preschoolers’ counting: Principles before skill. Cognition 13, 343-359. doi: https://doi.org/10.1016/0010-0277(83)90014$\underline{8}$

Gerstmann, J. (1940). The syndrome of finger agnosia, disorientation for right and left, agraphia and acalculia. Archives of Neurology and Psychiatry 44, 398-408. doi: https://doi.org/10.1001/archneurpsyc.1940.022 $\underline{80080158009}$

Ginsburg, H. \& Baroody, A. J. (1983). The test of early mathematics ability. Pro-ed. (adaptadores Nuñez y Lozano, 2007). Madrid: TEA Ediciones.

González-Castro, P., Rodríguez, C., Cueli, M., Cabeza, L. \& Álvarez, L. (2014). Competencias matemáticas y control ejecutivo en estudiantes con Trastorno por Déficit de Atención con Hiperactividad y Dificultades de Aprendizaje de las Matemáticas. Revista psicodidáctica, 19 (1), 125-143. doi: https://doi.org/10.1387/RevPsicodidact.7510

Gracia-Bafalluy, M. \& Escolano-Pérez, E. (2014). Aportaciones de la neurociencia al aprendizaje de las habilidades numéricas. Revista de neurología, 58(2), 69-76. doi: https://doi.org/10.33588/rn.5802.2013262

Grafman, J. (1988). Acalculia. In: Boller, F., Grafman, J., Rizzolatti, G., \& Goodglass, H. (eds.), Handbook of Neuropsychology (Vol. 1), Elsevier, Amsterdam, pp. 121-136.

Grégoire, J., Nöel, M-P. \& Van Nieuwenhoven, C. (2015). TEDI-MATH, Test para el diagnóstico de las Competencias Básicas en Matemáticas (2 ${ }^{\mathrm{a}}$ ed.). (Manuel, J. Sueiro y Jaime Pereña, adaptadores). Madrid: TEA Ediciones.

Gross-Tsur, V., Manor, O. \& Shalev, R. S. (1996). Developmental dyscalculia: Prevalence and demographic features. Dev. Med. Clin. Neurol. 38, 25-33. doi: https://doi.org/10.1111/j.1469-

8749.1996.tb15029.x

Gillum, J. (2014). Assessment with children who experience difficulty in mathematics. Support for Learning, 29(3), 275-291. doi: https://doi.org/10.1111/1467-9604.12061

Henschen, S. E. (1925). Clinical and anatomical contributions on brain pathology. Arch. Neurol. Psychiatry 13: 226-249. doi: https://doi.org/10.1001/archneurpsyc.1925.022 $\underline{00080073006}$

Jacubovich, S (2006). Modelos de procesamiento del número. Revista Argentina de Neurología, 7, 21-31.

Käser, T., Baschera, G., Kohn, J., Kucian, K., Richtmann, V., Grond, U., Gross, M. \& Von Aster, M. (2013). Design and evaluation of the computer-based training program Calcularis 
Benedicto-López, Patricia \& Rodríguez-Cuadrado, Sara (2019). Discalculia: manifestaciones clínicas, evaluación y diagnóstico. Perspectivas actuales de intervención educativa. RELIEVE, 25(1), art. $7 . \quad$ doi: http://doi.org/10.7203/relieve.25.1.10125

for enhancing numerical cognition. Front Psychol, 4, 489.4 doi: https://doi.org/10.3389/fpsyg.2013.00489

Kirk, S. A. \& Bateman, B. (1962). Diagnosis and remediation of learning disabilities. Exceptional Children. doi: https://doi.org/10.1177/001440296202900204 Koehler, O. (1951). The ability of birds to “count”. Bulletin of Animal Behavior, 9, 4145.

Kosc, L. (1974). Developmental dyscalculia. Journal of Learning Disabilities, 7, 164-177. doi:

https://doi.org/10.1177/002221947400700309

Kucian, K., Grond, U., Rotzer, S., Henzi, B., Schönmann, C., Plangger, F. \& Von Aster, M. (2011). Mental number line training in children with developmental dyscalculia. NeuroImage, 57(3), 782-795. doi: https://doi.org/10.1016/j.neuroimage.2011.01. $\underline{070}$

Langer, J. (1986). The Origins of Logic: One to Two Years. Academic Press, New York.

Leh, J. M., \& Jitendra, A. K. (2013). Effects of computer-mediated versus teacher-mediated instruction on the mathematical word problem-solving performance of third-grade students with mathematical difficulties. Learning Disability Quarterly, 36(2), 68-79. doi: https://doi.org/10.1177/0731948712461447

Ley 13/1982 de Integración Social del Minusválido (LISMI). (BOE, núm. 103, 30 de abril de 1982).

Ley Orgánica 1/1990, de 3 de octubre, de Ordenación General del Sistema Educativo. (LOGSE). (BOE núm. 238, 4 de octubre de 1990).

Ley Orgánica 2/2006 de 3 de mayo, de Educación (LOE). (BOE núm. 106, 4 de mayo de 2006).

Ley Orgánica para la mejora de la calidad educativa (LOMCE) (Ley Orgánica 8/2013, 9 de diciembre). Boletín Oficial del Estado, $\mathrm{n}^{\circ}$ 295, 2013, 10 diciembre.

Lewandowsky, M., \& Stadelmann, E. (1908). Ueber einen bemerkenswerten Fall von Himblutung und uber Rechenstorungen bei Herderkrankurg des Gehirns. J. Psychol. Neurol. 11, 249-265.

Lewis, C., Hitch, G. J. \& Walker, P. (1994). The prevalence of specific arithmetic difficulties and specific reading difficulties in 9 to 10 year old boys and girls. J. Child Psychol. Psychiatry 35, 283-292. doi: https://doi.org/10.1111/j.1469-

7610.1994.tb01162.x

Lievegoed, B. (1999). Las etapas evolutivas del niño. Madrid: Rudolf Steiner.

Loveland, K. A., Fletcher, J. M. \& Bailey, B. (1990). Verbal and nonverbal communications of events in learning disabilities subtypes. $J$. Clin. Exp. Neuropsychol. 12, 433-447. doi: https://doi.org/10.1080/01688639008400991

Manga, D. \& Campos, F. R. (1991). Neuropsicología de la edad escolar: aplicaciones de la teoría de AR Luria a niños a través de la Batería Luria-DNI. Visor.

Martínez Zamora, M., Henao López, G.C. \& Gómez, L.A. (2009). Comorbilidad del trastorno por déficit de atención e hiperactividad con los trastornos específicos del aprendizaje. Revista Colombiana de Psiquiatría, 38 (1), 178-194.

McCloskey, M., Caramazza, A. \& Basili, A. (1985). Cognitive mechanisms in number processing and calculation: Evidence from dyscalculia. Brain Cogn. 4, 171-196. doi: https://doi.org/10.1016/0278-2626(85)90069-7 Mechner, F. (1958). Probability relations within response sequences under ratio reinforcement. J. Exp. Anal. Behav. 1, 109-121. doi: https://doi.org/10.1901/jeab.1958.1-109

Millá, M.G. (2006). Atención temprana de las dificultades de aprendizaje. Revista de neurología, 42 (supl 2), 223-227. doi: https://doi.org/10.33588/rn.42S02.2005821

Miranda Casas, A., Meliá de Alba, A., Marco Taverner, R. Roselló, B. \& Mulas, F. (2006). Dificultades en el aprendizaje de matemáticas en niños con trastorno por déficit de atención e hiperactividad. Revista de neurología, 42 (supl 2), 163-170. doi: https://doi.org/10.33588/rn.42S02.2005788

Mora, J. \& Aguilera, A. (2000). Dificultades de aprendizaje y necesidades educativas especiales. En J. Mora \& A. Aguilera (Coords.) Atención a la diversidad en educación: Dificultades en el aprendizaje del lenguaje, de las matemáticas y en la socialización (p. 13-44). Sevilla: Kronos.

Needs, S. E. (1978). Report of the committee of enquiry into the education of handicapped 
Benedicto-López, Patricia \& Rodríguez-Cuadrado, Sara (2019). Discalculia: manifestaciones clínicas, evaluación y diagnóstico. Perspectivas actuales de intervención educativa. RELIEVE, 25(1), art. $7 . \quad$ doi: http://doi.org/10.7203/relieve.25.1.10125

children and young people. London: Her Majesty's Stationary Office.

Nelwan, M., Vissers, C., \& Kroesbergen, E. H. (2018). Coaching positively influences the effects of working memory training on visual working memory as well as mathematical ability. Neuropsychologia, 113, 140-149. doi: https://doi.org/10.1016/j.neuropsychologia.20 $\underline{18.04 .002}$

Olea, R., Ahumada, H. \& Líbano, L.E. (1986). Prueba de Aptitud y Rendimiento Matemático. República de Chile: CPEIP.

Palomino, A. \& Crespo, J. (2005). Prueba de Cálculo y Nivel Matemático.

Pérez, J. A. P., Arias, M. D. R. M. \& Mateos, R. M. (2000). Cuestionario de madurez neuropsicológica infantil, CUMANIN. TEA Ediciones.

Piaget, J. (1959). The language and thought of the child (Vol. 5). Psychology Press.

Portellano, J. A., Mateos, R. \& Martínez-Arias, R. (2012). Cuestionario de Madurez Neuropsicológica escolar (CUMANES).

Räsänen, P., Salminen, J., Wilson, A. J., Aunio, P. \& Dehaene, S. (2009). Computer-assisted intervention for children with low numeracy skills. Cognitive Development, 24(4), 450472. doi: https://doi.org/10.1016/j.cogdev.2009.09.003

Re, A. M., Pedron, M., Tressoldi, P. E., \& Lucangeli, D. (2014). Response to Specific Training for Students With Different Levels of Mathematical Difficulties. Council for Exceptional Children, 80(3), 337-352. doi: https://doi.org/10.1177/0014402914522424

Rebollo, M. \& Rodríguez, A.L. (2006). Dificultades en el aprendizaje de las matemáticas. Revista de neurología, 42 (extra 2), 135-138. doi: https://doi.org/10.33588/rn.42S02.2005787

Rourke, B. P. (1987). Syndrome of non verbal learning disabilities: The final common path of white matter disease dysfunction. Clin. Neuropsychol. 1, 209-234. doi: https://doi.org/10.1080/13854048708520056

Rourke, B. P. (1988). The syndrome of non verbal learning disabilities: Developmental manifestation in neurological diseases, disorders, and dysfunctions. Clin. Neuropsychol. 2, 293-330. doi: https://doi.org/10.1080/13854048808403271
Rourke B. P. (1993). Arithmetic disabilities, specific and otherwise: A neuropsychological perspective. Journal of Learning Disabilities, 26, 214-226. doi: https://doi.org/10.1177/002221949302600402

Santamaría, P., Arribas, D., Pereña, J. \& Seisdedos, N. (2005). EFAI: Evaluación factorial de las aptitudes intelectuales. TEA.

Sans, A., Boix, C., Colomé, R., López-Sala, A. \& Sanguinetti, A. (2012). Trastornos del aprendizaje. Pediatria Integral, 16(9), 691699.

Sans, A., López-Sala, A., Colomé, R., Boix, C. \& Sanguinetti, A. (2013). Trastornos del Aprendizaje. En AEPap ed. Curso de Actualización Pediátrica (p.37-47). Madrid: Exlibris Ediciones.

Serra-Grabulosa, JM. (2014). La discalculia. Dentro de Andreu i Barranchina, LL., et al. Trastorno de aprendizaje de la escritura y las matemáticas ( $1^{\mathrm{a}}$ ed., p.185-227). Barcelona: UOC.

Siegler, R. S. (1987). Strategy choices in subtraction. In: Sloboda, J. A., and Rogers, D. (eds.), Cognitive Processes in Mathematics, Clarendon Press, Oxford.

Shalev, R. S., Auerbach, J. \& Gross-Tsur, V. (1995). Developmental dyscalculia behavioral and attentional aspects: A research note. $J$. Child Psychol. Psychiatry, 36, 1261-1268. doi: $\quad$ https://doi.org/10.1111/j.14697610.1995.tb01369.x

Shalev, R., Weirtman, R. Amir, N. (1988). Developmental dyscalculia. Cortex 24, 555561. doi: https://doi.org/10.1016/S00109452(88)80049-2

Spreen, O., \& Strauss, E. (1991). A Compendium of Neuropsychological Tests. Oxford University Press, New York.

Strang, J. D. \& Rourke, B. P. (1985). Arithmetic disabilities subtypes: The neuropsychological significance of specific arithmetic impairment in childhood. In: Rourke, B. P. (ed.). Neuropsychology of Learning Disabilities, Guilford Press, New York, pp. 87-101.

Temple, C. (1997). Developmental cognitive neuropsychology. Hove: Psychology Press.

Thurstone, L. L. (1938). Primary mental abilities.

Thurstone, L. L. \& Thurstone, T. G. (2004). TEA. Test de Aptitudes Escolares. 
Benedicto-López, Patricia \& Rodríguez-Cuadrado, Sara (2019). Discalculia: manifestaciones clínicas, evaluación y diagnóstico. Perspectivas actuales de intervención educativa. RELIEVE, 25(1), art. $7 . \quad$ doi: http://doi.org/10.7203/relieve.25.1.10125

Vygotsky, L. (1931). Desarrollo de las funciones mnemónicas y mnemotécnicas. Lev Vygotsky Obras Escogidas, 3, 247-264.

Weschler, D. (2012). Escala de Inteligencia de Weschler para preescolar y primaria. WWPSI-IV. (Dpto de I+D Pearson Clinical and Talent Assessment, 2014). Madrid: TEA ediciones.

World Health Organization. (1992). ICD-10: International statistical classification of diseases and health-related problems. Geneva: WHO.

World Health Organization. (2018). ICD-11: International classification of diseases 11th Revision ICD-11 Beta Draft 2017.

Wilson, A.J., Dehaene, S., Pinel, P., Revkin, S.K., Cohen, L., \& Cohen, D. (2006). The principles underlying the design of "The Number Race” and adaptative computer game for remediation of dyscalculia. Behav. Brain Funct., $2, \quad 19 . \quad$ doi: https://doi.org/10.1186/1744-9081-2-19

Wynn, K. (1990). Children's understanding of counting. Cognition 36, 155-193. doi: https://doi.org/10.1016/0010-0277(90)90003-3 Wynn, K. (1992). Children's acquisition of the number words and counting system. Cognit. Psychol. 24, 220-251. doi: https://doi.org/10.1016/0010-0285(92)90008-P Yuste, C., Martínez, R. \& Galve, J. L. (2005). Batería de Aptitudes Generales y Diferenciales-Renovado (BADYG-R).

\section{Authors / Autores}

To know more / Saber más

\section{Benedicto-López, Patricia (patriciabenlop@gmail.com)}

Licenciada en Psicología por la Universidad de Murcia (intensificación en Psicología Clínica y Educativa). Máster en Dificultades de Aprendizaje y Trastornos del Lenguaje por la Universitat Oberta de Cataluña. Actualmente ejerce su labor como Profesora de Enseñanza Secundaria en la especialidad de orientación educativa en el Centro Público de Educación Especial Primitiva López. Sus líneas de trabajo se relacionan con las necesidades educativas especiales en alumnos gravemente afectados, dificultades de aprendizaje, sistemas alternativos y aumentativos de comunicación, estimulación multisensorial e intervención y tratamiento educativo de las necesidades específicas de apoyo educativo. Su dirección postal es: Área de Orientación educativa, CEE Primitiva López, C/ Serranía de Ronda, 1. CP: 30310, Cartagena, Murcia.

\section{Rodríguez-Cuadrado, Sara (sara.rodriguezcuadrado@uam.es)}

Licenciada en Psicología por la Universidad Autónoma de Madrid y doctora en Biomedicina (especialidad Neurociencia) por la Universidad Pompeu Fabra. Máster en Ciencia Cognitiva y Lenguaje por la Universidad de Barcelona y estudios de posgrado en Detección y Tratamiento de las Dificultades de Aprendizaje por la Universidad de Barcelona, y en Enseñanza en Educación Superior por Edge Hill University (Ormskirk, Reino Unido). Actualmente trabaja como profesora Ayudante Doctora en el Departamento Interfacultativo de Psicología Evolutiva y de la Educación de la Universidad Autónoma de Madrid (Madrid, España). Sus líneas de trabajo se relacionan con las dificultades de aprendizaje, el aprendizaje de segundas lenguas orales y de signos, el procesamiento del lenguaje emocional y la integración multisensorial. Su dirección postal es: Departamento Interfacultativo de Psicología Evolutiva y de la Educación-UAM Facultad de Formación del Profesorado y Educación, Despacho II-214 Universidad Autónoma de Madrid. C/ Francisco Tomás y Valiente, $n^{\circ}$ 3, 28049, Madrid 
Benedicto-López, Patricia \& Rodríguez-Cuadrado, Sara (2019). Discalculia: manifestaciones clínicas, evaluación y diagnóstico. Perspectivas actuales de intervención educativa. RELIEVE, 25(1), art. $7 . \quad$ doi: http://doi.org/10.7203/relieve.25.1.10125

\section{RELIEVE}

Revista ELectrónica de Investigación y EValuación Educativa E-Journal of Educational Research, Assessment and Evaluation

[ISSN: 1134-4032]

\section{(c) $(1)$ (9)}

Esta obra tiene licencia de Creative Commons Reconocimiento-NoComercial 4.0 Internacional. This work is under a Creative Commons Attribution 4.0 International license. 